\title{
DIFICULTADES DE APRENDIZAJE DEL CÁLCULO: CONTRIBUCIONES AL DIAGNÓSTICO PSICOPEDAGÓGICO
}

\section{DIFFICULTIES IN LEARNING CALCULATION: CONTRIBUTIONS TO THE PSYCHOPEDAGOGICAL ASSESSMENT}

\author{
Alejandra Balbi \\ Universidad Católica del Uruguay, Uruguay \\ Sergio Dansilio \\ Universidad Católica del Uruguay, Uruguay
}

\begin{abstract}
Resumen: Existe suficiente evidencia científica sobre la Discalculia del Desarrollo (DD) que describe los procesos cognitivos específicos del cálculo y procesos neuropsicológicos relacionados como la memoria de trabajo y percepción visoespacial. Sin embargo es llamativa su baja identificación diagnóstica en la infancia.

Este estudio tiene el objetivo de proporcionar evidencia clínica que contribuya a un diagnóstico riguroso. Se presentarán dos estudios de caso ( 7 y 8 años) que demuestran a través de pruebas normalizadas y de administración individual, déficits en la comprensión del número y la realización de cálculos elementales, en la percepción visoespacial, atención y memoria de trabajo. Se mostrarán los principales signos que permiten identificar el problema al inicio de la escolarización a efectos de realizar precozmente las orientaciones psicopedagógicas necesarias.

La inteligencia es normal de acuerdo a pruebas estándar, la instrucción educativa es adecuada, y se descartan alteraciones sensoriales y emocionales. Estos hallazgos contribuyen en la descripción adecuada de la semiología esperada ante una DD para un adecuado diagnóstico. En los casos descritos, se encuentran perturbaciones visuoespaciales posiblemente relacionadas.
\end{abstract}

Palabras clave: Discalculia del desarrollo, memoria de trabajo, procesos neuropsicológicos, comprensión del número.

\begin{abstract}
There is enough empirical evidence about developmental calculation disorders (DCD), where specific impairments in basic mathematical operations and related cognitive systems as working memory and perception have been described.

This paper aims to provide clinical evidence contributing to establish a rigorous diagnosis in the mentioned domain. A two-case study is presented ( 7 and 8 years old children). Numerical comprehension and basic operation deficit is shown in individually applied validated batteries, as well as in working memory and perception domains. Main diagnostic indicators of the cognitive disturbance in early schooling are described. Early identification is essential to the opportune pedagogical approach.

There is no general intelligence, sensorial or emotional impairments in any of the studied cases. These findings provide additional clinical resources for clinicians facing a DCD. In the present cases, a visuospatial disorder could be related to the deficit.
\end{abstract}

Keywords: Developmental dyscalculia, working memory, neuropsychological processes, number comprehension.

\section{INTRODUCCIÓN}

Existe consenso en considerar la Discalculia del Desarrollo (de ahora en adelante DD) como un disturbio heterogéneo en la competencia numérica y del cálculo matemático, en sujetos con escolaridad adecuada, ausencia de retraso intelectual, déficit sensorial o trastornos emocionales. (Temple, 1991; American Psychiatric
Association, 1995; Dansilio, 2008b) Se informa de una prevalencia de $3 \%$ a $6 \%$. Puede asociarse a la dislexia pero constituye un trastorno disociable de ésta. (Shalev, Auerbach, Manor, Gross-Tsur, 2000). La DD supone dificultades en la comprensión de conceptos numéricos simples, problemas para recuperar cálculos y utilizar procedimientos para resolverlos. Incluso si llegaran a responder u utilizar un procedimiento

Correspondencia: Alejandra Balbi. Facultad de Psicología, Universidad Católica del Uruguay.

Correo Electrónico: abalbi@ucu.edu.uy. 
correcto, pueden hacerlo mecánicamente y sin confianza (Gifford, 2005).

Las discalculias no se presentan como una entidad única y simple sino que son heterogéneas y de gran variablilidad, lo que dificulta su adecuado diagnóstico (Dowker, 1998). Niños con o sin discalculia pueden tener diferentes áreas fuertes y débiles en diferentes dominios de la matemática: cálculo, recuerdo de hechos numéricos, conocimientos conceptuales de las operaciones, resolución de problemas. Ginsburg (1977), recomienda complementar los datos recogidos en los tests con una entrevista clínica en profundidad para lograr un conocimiento más acabado de la variabilidad en esas áreas fuertes y débiles de cada niño, sus errores y las estrategias para sortearlos. Es decir, incorporar rigurosamente el análisis cualitativo a las evidencias proporcionadas por los test.

Un diagnóstico de Discalculia debe cumplir con los criterios de exclusión propuestos por el DSM IV y demostrar criterios de discrepancia y persistencia utilizando pruebas normalizadas y de administración individual (1995). Se utilizan diferentes procedimientos para establecer el criterio de discrepancia pero existe consenso en considerar más de dos desvíos estándar por debajo del desempeño esperado a su edad cronológica. El criterio de persistencia es esencial dada la heterogeneidad y variabilidad de los rendimientos. En un estudio prospectivo longitudinal durante seis años (Shalev, Manor \& Gross-Tsur, 2005) informan que la DD constituye una dificultad de aprendizaje permanente que persiste en la adolescencia tardía en casi la mitad de las personas identificadas inicialmente. Andersson (2010) ratifica la persistencia en la adolescencia de la DD, así como su efecto desfavorable en dominios cognitivos generales.

Los diagnósticos presuntivos posibilitan iniciar intervenciones psicopedagógicas de acción temprana e intensiva en los primeros años de escolaridad discriminando de esta forma al grupo de sujetos con rendimiento débil en cálculo, de aquellos con DAC. Butterworth, B., Reigosa, V. (2007) proponen utilizar el término Dificultades de Aprendizaje de la Aritmética para describir a los niños con el 30\% más bajo y Discalculia del Desarrollo al grupo más severo que las estimaciones de prevalencia sitúan entre 3,6 \% y 6,4 \%.
La comorbilidad con otras disfunciones es elevada, Gross-Tsur (1996) realizó un estudio de prevalencia de Discalculia, donde además de ratificar la prevalencia de un $6,2 \%$ discrimina en este grupo, un $17 \%$ con dificultades lectoras (DAL) y un 26\% Déficit Atencional. Shalev, Auerbach y Gross Tsur (1995) analizan mediante el Child Behavior Checklist la incidencia de factores emocionales asociados a DAC, identificando relaciones de valor clínico con el déficit atencional, aún con valores normales para las escalas de ansiedad y depresión.

Cumplidos los criterios de exclusión, discrepancia y persistencia, el clínico debe estar entrenado en identificar lo que las evidencias señalan como tres aspectos consistentes en los sujetos con DD: (1) la persistencia de estrategias inmaduras de conteo, (2) la dificultad en la recuperación de hechos numéricos (HNB) almacenados en la memoria y (3) la dificultad para resolver problemas que involucran múltiples pasos (Andersson, 2010, Gifford, 2005, Geary, 2003, 2004; Butterworth, Lander et al, 2003; Ostad, 1999, Vukovic \& Siegel, 2010).

A medida que el niño avanza en sus primeros años de escolaridad, acumula experiencias de conteo y numeración que posibilitan consolidar lo que Butterworth (2005) llama sentido numérico. Es así que puede estimar y comparar la magnitud de un número con otro sin necesidad de recitar la serie numérica, realiza estrategias de descomposición aditiva para obtener un cálculo (para sumar 8+7 puede llegar al $10 y$ luego sumar lo que le queda...") o sabe que el "siete" puede formarse de muchas maneras: $6+1,5+2$, etc. El desarrollo normal del sentido numérico muestra que hacia los 8 o 9 años el sujeto prescinde progresivamente del conteo basado en los dedos o el recuento verbal para apelar a la recuperación de cálculos almacenados en su memoria a largo plazo. Son llamados Hechos Numéricos Básicos, de aquí en más HNB (Baroody, 1988). Recuperar los cálculos de la memoria permite mayor agilidad y una mejor administración de los recursos cognitivos, al prescindir del conteo cada vez que debe calcular, pudiendo acceder a procesos más complejos de la matemática.

Geary (2004) sugiere que los niños con DD recuperan los HNB con mayor lentitud, cometen errores procedimentales y persisten en el 
empleo de estrategias inmaduras como volver a contar todo y sobrecontar (Brissiaud, 1986). A pesar de recibir intervención especializada, continúan requiriendo de contar con los dedos, cuando sus pares sin DD recuperan HNB de su memoria a largo plazo (Landerl, Butterworth, 2004). Esta dificultad constituye otro de los indicadores diagnósticos específicos, ya que la misma persiste, a pesar de la intervención (Ostad, 1997). A su vez, en este estudio longitudinal realizado en sujetos noruegos de $1^{\circ}, 3^{\circ}$ y $5^{\circ}$ año escolar se evidenció que el procedimiento de los sujetos con DD además de lento y engorroso, era ineficaz, ya que los errores eran altamente frecuentes.

La relación entre estos procesos específicos del cálculo y los dominios neuropsicológicos de memoria de trabajo, atención y organización visoespacial es una fuente de profusa investigación en la actualidad.

Miranda et al, (2006) investigaron las relaciones entre memoria de trabajo verbal y visoespacial y Dificultades de Aprendizaje del Cálculo (DAC) en sujetos entre 6 y 13 años con y $\sin$ TDAH. Diferenciaron cuatro grupos: DAC, DAC+TDAH, TDAH y grupo control indicando que los tres primeros mostraban resultados significativamente más bajos al grupo control en tareas de recuerdo de dígitos inverso y de recuerdo visoespacial. Aunque puntualizan que el descenso en memoria de trabajo visoespacial solo se observa en los dos grupos con DAC, por lo que se discute que podría ser uno de los mecanismos subyacentes en los sujetos con DAC con independencia de su condición de TDAH.

Gerstmann (1942) ha relacionado una serie de síntomas que incluyen agnosia digital, agrafia, indiferenciación derecha e izquierda y acalculia. Dansilio (2008a) presenta evidencia de tres casos que cumplen con los elementos fundamentales del síndrome de Gerstman del desarrollo (agnosia digital, indistinción derecha/ izquierda, discalculia), mostrando afectando el dominio visuoconstructivo en uno de ellos. Otros trabajos han relacionado trastornos del cálculo con perturbaciones de la coordinación visomotora y el dominio psicomotriz en general, así como disturbios a nivel del período sensoriomotor piagetiano (Yeo, 2003; Rourke, 1993) Dehaene (2001) propuso que el modo básico para representar números en el cerebro es por una especie de línea numérica mental, que es esencialmente espacial. Esto proporciona las imágenes de magnitud aproximada, mientras que la aritmética exacta depende de un sistema predominantemente verbal. Kaufman, L. (2008) aporta evidencia que relaciona la gnosia digital con el dominio del cálculo en la etapa escolar, sugiriendo implicaciones educativas para estimular el conteo digital en su adquisición. Rotzer, et al (2009) revisan la relación entre memoria de trabajo espacial y discalculia, apoyando la hipótesis de que una pobre capacidad de memoria de trabajo puede dificultar la adquisición de las representaciones espaciales del número de niños con DD.

Sin embargo, las relaciones entre las DAC y los diferentes sistemas de memoria de trabajo, es un tema de controversia (Gevers, W., Santens, S., Dhooge, E., Chen, Q., 2010). Alsina y Saiz (2003) y posteriormente Alsina (2007) en un estudio dirigido a analizar el papel del ejecutivo central, el bucle fonológico y la agenda visoespacial en niños españoles con DAC, encuentran únicamente correlación entre el ejecutivo central y el bucle fonológico con las DAC, y no con la agenda visoespacial.

Los diferentes reportes avanzan en la línea de que el ejecutivo central sería el dispositivo de la memoria de trabajo que mejor se relaciona, por sobre el bucle fonológico y la agenda visoespacial. (Mc Lean \& Hitch, 1999; Siegel \& Ryan, 1989; Bull, 1999). El ejecutivo central tendría un papel relevante en sostener y monitorizar los problemas aritméticos que requieren múltiples pasos, inhibiendo información mientras se procesa información nueva o recuperando el dato almacenado para integrarlo con el nuevo dato obtenido (Russel, Ginsburg, 1984). En esta línea, Miranda (2009) aporta evidencia que indicaría que el déficit en funciones ejecutivas agravaría el rendimiento en matemática de forma más severa que en lectura.

Algunas investigaciones señalan que los sujetos con DAC suelen obtener puntuaciones normales en tareas de recuerdo verbal pero puntuaciones bajas en tareas de recuerdo numérico (Siegel \& Ryan, 1989, Mac Lean \& Hitch, 1999). Ello parecería sugerir que el problema se debe a una dificultad específica para mantener la información numérica en la memoria de trabajo. 
Apoyan esta tesis la disociación anatómica y funcional de estos dos sistemas de memoria en distintas zonas del cerebro (Thioux, Seron \& Pesenti, 1999), aunque esta hipótesis aún requiere evidencias más sustantivas.

\section{ESTUDIO DE CASOS}

Se presentan casos de dos niños que serán llamados Martín (8,0 años) y Maia (7,3 años). Ambos llegan a la consulta psicopedagógica y se realiza evaluación que incluye administración de pruebas individuales y normalizadas: WiscIII, Prolec-R, Pro-Cálculo, Bender, pruebas criteriales en escritura y aritmética, entrevista familiar, historia escolar y médica.

No hay antecedentes patológicos prenatales ni perinatales. El desarrollo en los primeros años de vida es normal según historia médica. Se excluyen problemáticas afectivas de entidad y déficits sensoriales. El ambiente familiar y socio educativo es favorable.

\section{Caso 1}

Martín es derivado desde la institución educativa a consulta psicomotriz y médica por distractibilidad al cursar la última etapa de educación inicial. Se indica estudio psicológico que informa sobre buena capacidad intelectual y ausencia de trastornos emocionales severos. Ante indicadores de disgrafía se inicia tratamiento psicomotriz con alta frecuencia durante 2 años consecutivos. A los 6 años, ante la persistencia de la distractibilidad, se realiza nueva consulta médica quien realiza diagnóstico presuntivo de TDAH y solicita estudio psicopedagógico. Se inicia tratamiento farmacológico con Metilfenidato. Su historia escolar, el estudio psicopedagógico destaca que más allá de la persistencia de una disgrafía resistente a la intervención Martín aprende idiomas con facilidad y asimila información relacionada con ciencias sociales o naturales de forma adecuada. Sin embargo el aprendizaje de la lectura, escritura y aritmética se desarrolla con dificultad. Ambos padres son profesionales. Se reportan dificultades en la concentración en varios miembros del grupo familiar.

\section{Caso 2}

Maia es derivada a la consulta psicopedagógica por la institución educativa al comenzar $2^{\circ}$ año escolar (8 años) para indagar específicamente sus dificultades en aritmética. En anamnesis con padres se informa de un desarrollo neuropsicológico y afectivo normal, sin dificultades en los aprendizajes relacionados con la lengua oral y escrita. Fue promovida con buena calificación en su primer año escolar. Sus padres observan que Maia progresa lentamente en el área de matemática, y optaron por iniciar apoyo extraescolar durante primer año escolar. En $2^{\circ}$ año escolar, cambia de instituciones escolar, donde se evalúa la pronta necesidad de indagar sus dificultades en matemática. Los padres, ambos profesionales no reportan antecedentes de dificultades de aprendizaje.

A continuación se presentará la información seleccionada relevante para el diagnóstico psicopedagógico el cual recoge datos sobre: (1) ambiente familiar y escolar (2) desarrollo neuropsicológico, afectivo y cognitivo (3) capacidad intelectual, (4) procesos cognitivos básicos o neuropsicológicos de Lenguaje, Memoria de Trabajo y Organización Visoespacial, procesos cognitivos específicos implicados en (3) Lectura, Escritura y (4) Aritmética, (5) aprendizajes curriculares.

La Figura 1 ilustra puntajes tipificados que informan sobre rendimiento en procesos cognitivos neuropsicológicos o básicos tomando como referencia la Escala de Inteligencia para Niños WISC III. Dicha escala informa sobre ausencia de retraso intelectual, diferencia significativa entre funcionamiento verbal y visoespacial en la línea de los hallazgos mencionados (Rourke, 1993; Miranda, 2006, Rotzer et al, 2009; Dehane, 2001). No se informan resultados de $\mathrm{Cl}$ total ya que este valor debe desestimarse por la diferencia entre Escala Ejecutiva y Verbal. El Lenguaje evaluado en componentes de conceptualización, razonamiento y abstracción verbal a través del Índice de Comprensión Verbal (WISC III) refleja una fortaleza cognitiva en ambos sujetos, especialmente en el caso de Martín, donde demuestra un desempeño casi 2 DS por encima de la media poblacional.

EI IAD como puede apreciarse en la Figura 1 muestra un descenso próximo a 1 DS dado que este índice se compone por el subtest de Aritmética y el de Retención de Dígitos (RD). Se profundiza el análisis al poder discriminar el recuerdo de dígitos directo del recuerdo de 


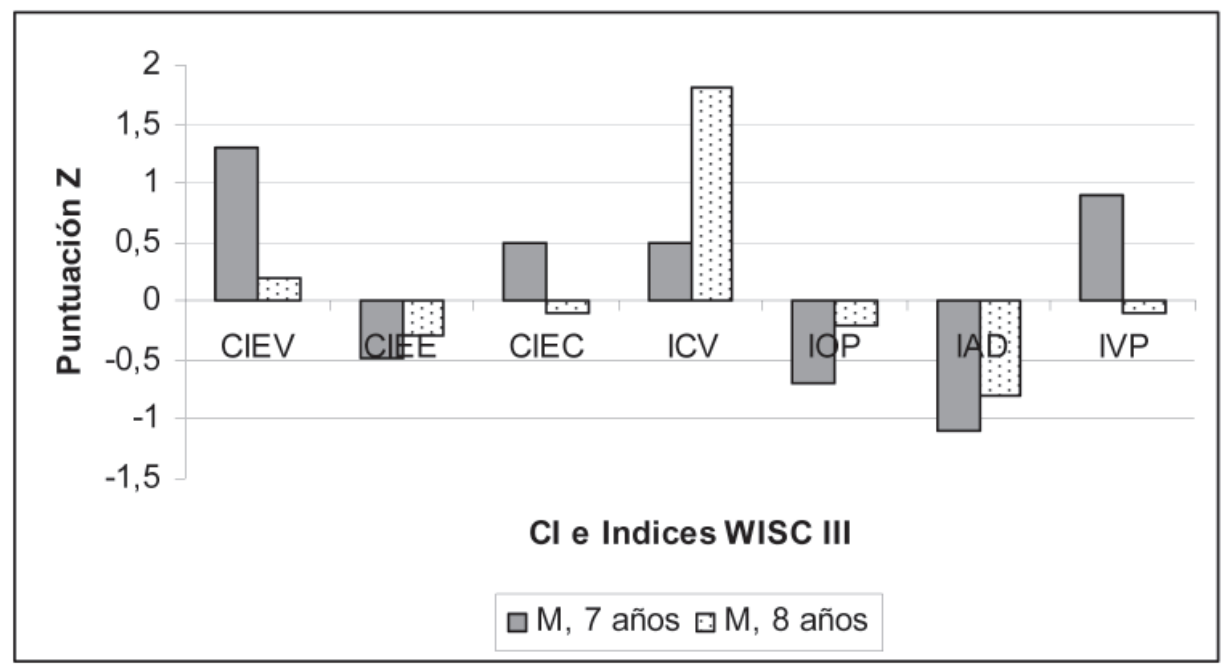

Figura 1. Nivel Intelectual y procesos cognitivos básicos

dígitos inverso, ya que la literatura relaciona a Dígitos Directo (DD) con una memoria más pasiva y Dígitos Inverso (DI) con memoria de trabajo. Los resultados representados en la Figura 2 son elocuentes de la diferencia en ambos sujetos entre sostener información de forma pasiva a sostener y procesar activamente la información. El instrumento elegido de evaluación (retención de dígitos) exige considerar aportes de algunos investigadores (Siegel \& Ryan, 1989, Mac Lean \& Hitch, 1999) referentes a la existencia de un almacén específico para la información numérica. Es decir, resta analizar si la debilidad evidenciada respondería a un déficit de memoria de trabajo de forma genérica o a un déficit específico cuando se procesa información numérica.
Se estudia también la coordinación visomotora y la grafomotricidad propia de la escritura. La figura 3 muestra el desempeño en Bender quien muestran retrasos de valor diagnóstico. A su vez, lo que en Maia resultan indicadores de una disgrafía de leve entidad en Martín constituye una clara disgrafia, aún luego de dos años de tratamiento psicomotriz. Estos hallazgos podrían formar parte de la tétrada de Gertsman así como las evidencias de Yeo (2003) y Dansilio (2008b) en cuanto a la asociación entre DAC y trastornos de la psicomotricidad.

La evaluación de los procesos lectores demuestra en ambos sujetos desempeños con variaciones normales que permiten descartar Dificultades Lectoras.

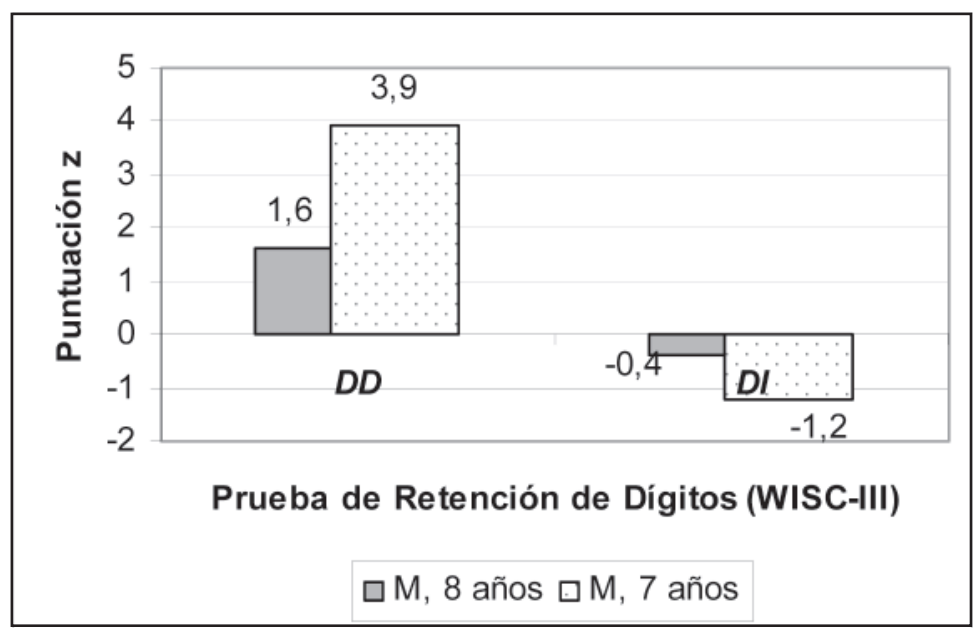

Figura 2. Tipificación discriminada entre Dígitos Directo y Dígitos Inverso (WISC III) 


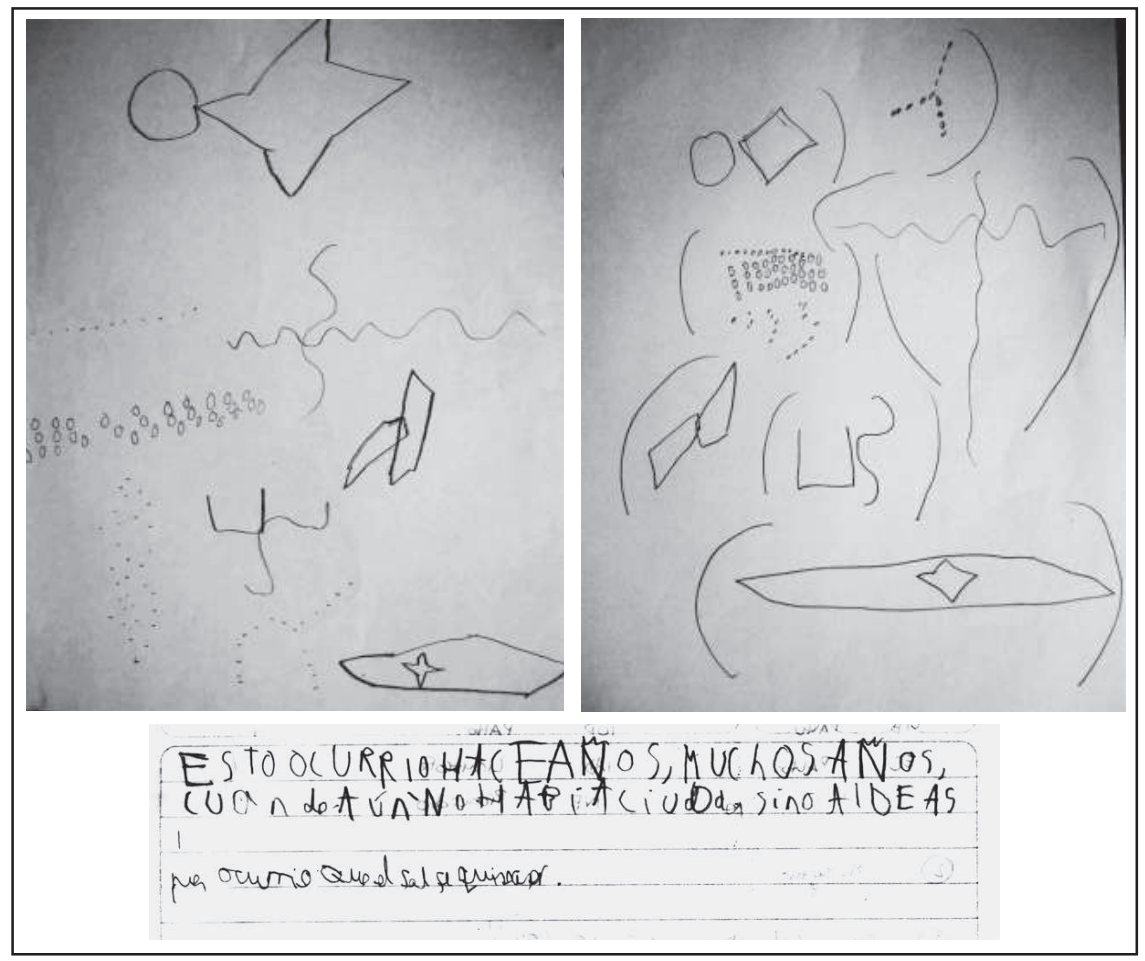

Figura 3. Coordinación visomotora y grafomotricidad

En la Figura 4 se muestra el desempeño en Lectura evaluado a través de Prolec- $R$ (2007). En Lectura de Pseudopalabras Maia muestra bajo rendimiento pero de acuerdo a la valoración clínica, no reviste significatividad. Se observa una estrategia poco analítica e impulsiva para leer pseudopalabras, aspecto que mejora rápidamente con la intervención. El desempeño en Comprensión Lectora se sitúa en ambos sujetos por encima del Promedio. Martín evidencia un destacado desempeño en Comprensión Lectora asociado con la capacidad en razonamiento verbal evidenciada a través del ICV (WISC III).
La perfomance reflejada por ambos sujetos en Comprensión Lectora refleja destrezas desatacadas en habilidades inferenciales. Estas sugieren buena capacidad intelectual y son indicadores de un mejor pronóstico en lo que respecta a rendimiento académico.

La evaluación de Aritmética se basa en técnicas cualitativas de: entrevista docente, análisis de resultados escolares y técnicas cuantitativas: Test Pro-Cálculo (2006), subtest de Aritmética de la Escala Weschler. Los resultados tipificados de las técnicas cuantitativas se pueden apreciar en la figura 5 .

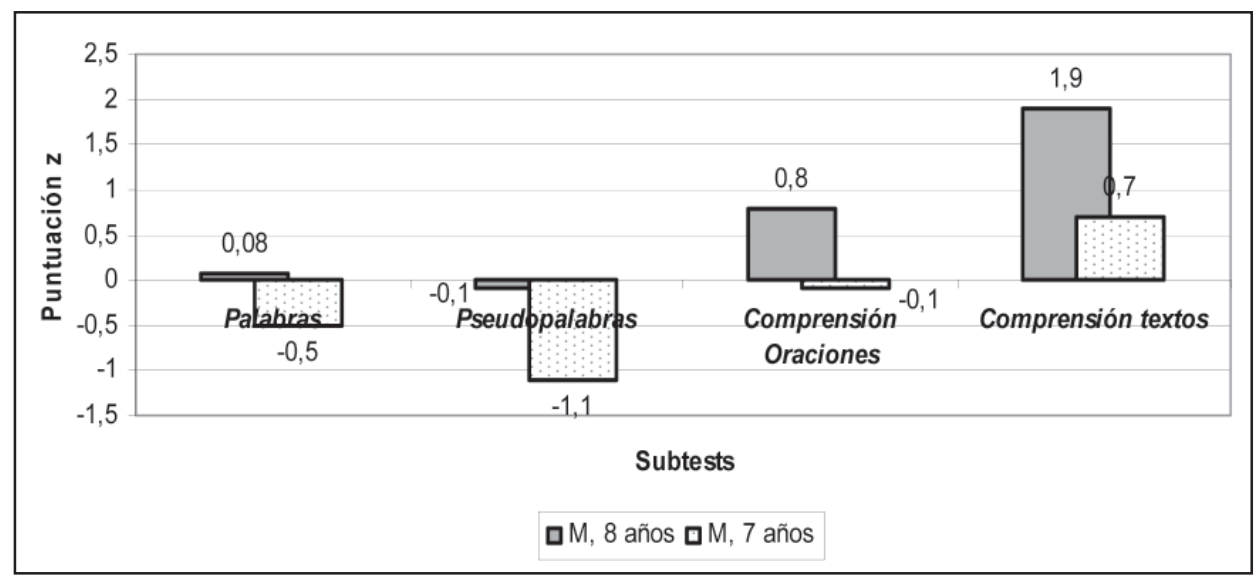

Figura 4. Desempeño en lectura (Prolec-R) 


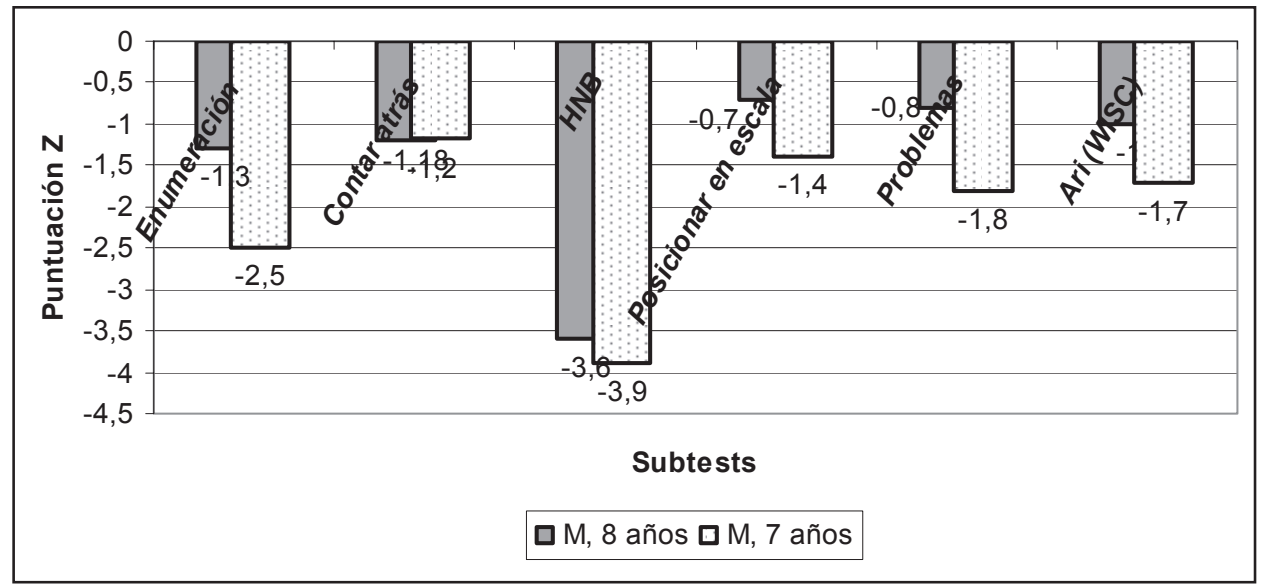

Figura 5. Desempeño en Matemática (Pro-Cálculo y WISC-III)

Los dos primeros subtest (Enumeración y Contar para Atrás) evidencian las estrategias inmaduras de conteo. Los sujetos muestran dificultad para cumplir con indicadores de conteo (Gelman \& Gallistel, 1978) de correspondencia y de cardinalidad. Los errores más frecuentes observados se producen por desorganización al reiterar objetos ya contados, o por fallas en la monitorización al "perder la cuenta" cuando la configuración espacial de los objetos exige detenerse para planificar la secuencia. Ante esto, en ocasiones, se observa la decisión de volver a contar, pero en otras, la fatiga y la falta de confianza en poder realizarlo mejor, limita un nuevo intento.

Los resultados en el subtest de HNB sitúan a ambos sujetos en un nivel de severidad que exime de mayor explicación. Desde el análisis de las estrategias empleadas por los sujetos podemos aportar que la ausencia de HNB, remite a la puesta en marcha de estrategias ineficaces, o en casos más extremos, a la ausencia de estrategias. Maia evidencia desconocer un procedimiento a partir del cual una situación inicial con dos cantidades simples para los que alcanzarían los dedos de la mano, pueda transformarse a través de un operador en otra situación final.

Es así que cuando el entrevistador proporciona ayudas gráficas específicas para andamiar en la resolución, las acciones de Maia reflejan únicamente conceptualizaciones estéticas y visuales de las cifras: las enlaza para formar un nuevo signo, las decora.
En cambio Martín, intenta operar utilizando recursos auxiliares como la recta numérica. Se observa que alterna entre estrategias de sobreconteo y otras más primitivas como volver a contar todo (Brissiad, 1986).

Con respecto a la Resolución de Problemas, hay variaciones importantes entre un sujeto y otro. Martín identifica la naturaleza del problema, así como las operaciones requeridas para solucionarlo. Sus fallas se producen en la realización de los algoritmos, que involucran problemas de múltiples pasos (Gifford, 2005). Retener un resultado en su memoria de trabajo para asociarlo a un nuevo cálculo se constituye en un obstáculo insalvable.

Maia, a pesar de su adecuado desarrollo lingüístico y buena comprensión de textos, no puede identificar la operación requerida. Utiliza sus fortalezas verbales explicando la argumentación del problema, pero no puede proponer una operación que se vincule con la situación problema.

\section{DISCUSIÓN Y CONCLUSIONES}

Esta presentación tiene por objetivo proporcionar evidencia clínica al diagnóstico psicopedagógico de Discalculia del Desarrollo (DD) que permita la identificación de necesidades educativas especiales de forma temprana.

Los casos presentados cumplen los criterios exclusión de ausencia de retraso intelectual, 
alteraciones sensoriales, emocionales y adecuado ambiente escolar y familiar. Con respecto a los procesos específicos involucrados en el cálculo, ambos sujetos muestran déficits en la comprensión del número y la realización de cálculos elementales. Su perfil de rendimiento matemático es heterogéneo como lo describe la literatura (Dowker, 2004), sujetos con DD pueden presentar diferentes puntos fuertes y débiles en su propio perfil de rendimiento matemático. Andersson (2010) describe los cuatro componentes afectados en los sujetos con discalculia: hechos numéricos, conceptos, procedimientos, y resolución de problemas, concluyendo que diferentes sujetos pueden mostrar variabilidad en los mismos. Nuestros casos aportan evidencia en este sentido, Martín muestra afectado únicamente el componente de Hechos Numéricos (HN) y Procedimiento, con buena capacidad de Resolución de Problemas y Conceptos. Maia, en cambio, muestra afectados los cuatro componentes.

Si bien la literatura no termina de ser concluyente al documentar la relación entre funciones visoespaciales y DD (Gevers et al. 2010), los dos casos presentados, documentan dicha correlación teórica. Vukovic, R.\& Siegel, L. (2010) tampoco identifica relación entre memoria de trabajo visoespacial y DD, pero ante la profusa evidencia que las asocia, proponen que dicha relación, puede constituir uno de los subtipos de DD.

Al discutir sobre las razones del subdiagnóstico, identificamos como una fuente de explicación la afectación intensa en las funciones visoespaciales. Una discrepancia significativa verbal-no verbal es comúnmente atendida y orientada hacia servicios de asistencia psicomotriz. Dado que esta dificultad es severa y persistente, los sujetos continúan necesitando asistencia psicomotriz dejando encubierta la semiología de la DD identificable a través del diagnóstico psicopedagógico. Subrayamos la importancia del rol docente que según reportan nuestros casos, fueron agentes claves de la detección y derivación temprana. En cambio se observó en los servicios de salud procedimientos diagnósticos que no incluyeron la hipótesis de la DD y por tanto, dilataron su identificación.

En la revisión realizada por Dowker (2004) referente a las acciones educativas y psicopedagógicas indicadas para las $\mathrm{DD}$, hace mención enfática a las oportunidades de cambio y pro- greso, con intervenciones tempranas, intensivas e individualizadas. No estaremos en condiciones de ofrecer esta respuesta mientras no realicemos diagnósticos apropiados. Compartimos con Dowker (2004) como otra explicación del subdiagnóstico, su preocupación referente a la fragmentación de la abundante evidencia científica referente a la DD. Las neurociencias, la psicología educativa y la didáctica de las matemáticas avanzan en líneas de investigación paralelas, lo cual limita un real aprovechamiento de la misma en ámbitos educativos. Apostamos a que la psicopedagogía en su carácter aplicado y transdisciplinar contribuya ante la necesidad de poner la investigación al servicio de una respuesta educativa temprana, preventiva y por tanto compensadora de las necesidades de los alumnos.

\section{REFERENCIAS}

Alsina, A. (2007). ¿Por qué algunos niños tienen dificultades para calcular? Una aproximación desde el estudio de la memoria humana. Revista Latinoamericana de Investigación en Matemática Educativa, 10 (3):315-333.

Alsina, A., \& Saiz, D. (2003). Un análisis comparativo del papel del bucle fonológico versus la agenda viso-espacial en el cálculo en niños de 7-8 años. Psicothema, 15 (2): 241-246.

American Psychiatric Association (1995). Diagnostic and statistical manual of mental disorders, (4 $4^{\mathrm{a}}$ ed.). Washington DC, EEUU: American Psychiatric Association.

Balbi, A. (2008). Dificultades del Cálculo en la Clínica Psicopedagógica. Poster presentado en Congreso Internacional de Neuropsicología, Buenos Aires, Argentina.

Baroody,A. (1988). Elpensamiento matemático de los niños. Un marco evolutivo para maestros de preescolar, ciclo inicial y educación especial. Madrid: Aprendizaje Visor.

Bermejo, V. (2004). Cómo enseñar matemáticas para aprender mejor. Madrid: Editorial CCS.

Brissiaud, R. (1986). El aprendizaje del cálculo en los niños. Madrid: Visor.

Bull, R.; Johnston, R., \& Roy, J. (1999). Exploring the Roles of the Visual-Spatial Sketch Pan and Central Executive in Childrens Arithmetical Skills: view from cognition and developmental neuropsychology. Developmental Neuropsychology, 15 (3), 421-442.

Butterworth, B. (2005). Developmental dyscalculia. En: Jamie ID Campbell (Ed), The Handbook of Mathematical Cognition (pp. 455-467). New York: Psychology Press.

Butterworth, B., \& Reigosa, V. (2007). Information processing deficits in dyscalculia. In Why is math so hard for some children? The nature and origins of mathematical learning difficulties and disabilities. (pp. 65-81): Baltimore, MD, US: Paul H Brookes Publishing. 
Cuetos, F., Rodriguez, B. \& Ruano, E. (2007). Prolec-R: Batería de Evaluación de los procesos lectores. Barcelona, Tea Ediciones.

Dansilio, S. (2008a). Síndrome de Gerstmann del Desarrollo y trastornos en la adquisición de las matemáticas. Ciencias Psicológicas, II (1), 55-64.

Dansilio, S. (2008b). El procesamiento del número y el cálculo: Las discalculias y las acalculias. Prensa Médica Latinoamericana: Montevideo.

Dehaene, S. (2001). Precis of the number sense. Mind and language, 16 (1), 16-36.

Dowker, A. (2004) What works for children with Mathematicals Difficulties? (Research Report, $n^{\circ} 554$ ). University of Oxford, Department for Education and Skills.

Dowker, A. (1998). Individual differences in normal arithmetical development. En Donlan, Ch. The development of mathematical skills. Hove: Psychology Press.

Feld V., Tausikk, I., Azaretto, C. (2006). Test para la evaluación del procesamiento del número y el cálculo en niños. Argentina: Paidós.

Gelman, R. \& Gallistel, CR (1978). The child's understanding of number. Cambridge, MA: Harvard University Press.

Gerstmann, J. (1940). Síndrome of finger agnosia, disorientation for right and left, agraphia and acalculia. Archives of Neurology and Psychiatry, 44, 398-408.

Geary, D. (2003). Learning disabilities in arithmetic: Problem solving differences and cognitive deficits. En Swanson, H. L.; Harris, K. \& Graham, S. (Eds.), Handbook of learning disabilities. New York: Guilford Press.

Geary, D. (2004). Mathematics and Learning Disabilities. Journal of Learning Disabilities, 37 ( 1), 4-15.

GeversW., Santens S., D'Hooge E., Chen Q., Van den Bossche L., Fias W. and Verguts T. (2010). Verbal-spatial and visuo-spatial coding of number-space interactions. Journal of Experimental Psychology: General, 139(1), 180-190.

Gifford, S. (2005). Young childrens difficulties in learning mathematics. Review of research in relation to dyscalculia. (Research Report, $n^{\circ}$ 05/1545) Roehampton University of Surrey, Qualifications and curriculum authotity.

Ginsburg, H.P. (1972). Children's knowledge and individualized instruction. Educational Technology, 8-12.

Ginsburg, H.P. (1977). Children's Arithmetic: How They Leam It and How You Teach It. New York, N.Y: Teachers' College Press.

Gross-Tsur, V., Manor, O., \& Shalev, R. (1996). Developmental dyscalculia: prevalence and demographic features. Developmental Medicine and Child Neurology, 38, 25-33.

Jordan, N.C., Hanich, L.B.,\& Kaplan, D. (2003). Arithmetic fact mastery in young children: a longitudinal investigation. Journal of Experimental Child Psychology, 85, 103-119.
Landerl K., Bevan, A., \& Butterworth, B. (2004) Developmental dyscalculia and Basic numerical capacities: a study of 8-9 year-old student. Cognition, 93 (2004), 99-125.

McLean, J.F., \& Hitch, G.J. (1999). Working memory impairments in children with specific arithmetic learning difficulties. Journal of Experimental Child Psychology, 74 (3), 240-260.

Miranda, A., Melia, A., Marco, R., Rosello, B., \& Mulas F. (2006). Dificultades en el aprendizaje de matemáticas en niños con trastorno por deficit de atención con hiperactividad, Simposio Satélite de Dificultades de Aprendizaje. Revista de Neurología; 42 (2):163-170.

Ostad, S. (1997). Developmental differences in addition strategies: A comparison of mathematically disabled and mathematically normal children. British Journal of Educational Psychology, 67, 345-357.

Ostad, S. (1999). Developmental progression of subtraction studies: a comparison of mathematically normal and mathematically disabled children. European Journal of Special Needs Education, 14 (1), 21-36.

Rourke, B. P. (1993). Arithmetic disabilities, specific and otherwise: A neuropsychological perspective. Journal of Learning Disabiilties, 26, 214-226.

Rourke, B. P., \& Conway, J. (1997). Neurological and neuropsychological dimensions of disabilities in arithmetic and mathematics. Journal of Learning Disabilities, 30, 34-46.

Russell, R. L., \& Ginsburg, H. P. (1984). Cognitive analysis of chîldren's mathematics difficulties. Cognition and Instruction, 21, 7-2.

Shalev, R., Auerbach, J., Manor, O., \& Gross-Tsur, V. (2000). Developmental Discalculia: prevalence and prognosis. European Child \& Adolescent Psyquiatry. 9:II/58-II/64.

Siegel L., \& Ryan (1989). The development of working memory in normally achieving and subtypes of learning disabled children. Child Development, 60, 973 980.

Temple, C. M. (1991). Procedural dyscalculia and number fact dyscalculia: Double dissociation in developmental dyscalculia. Cognitive Neuropsychology, 8, 155-176.

Thioux, M., Seron, X., \& Pesenti, M. (1999). Functional neuroanatomy of the semantic system: The case for numerals. Brain and Language, 69, 488-490.

Yeo, D. (2003). Dyslexia, Dyspraxia and Mathematics. London: Whurr.

Weschler, D. (2002) Escala de Inteligencia para Niños WISC III. Argentina: Paidós. 